\title{
PARTIAL NEPHRECTOMY OF A HORSESHOE KIDNEY WITH RENAL-CELL CARCINOMA AND CHOLECYSTECTOMY: A CASE REPORT
}

\author{
Elif Cengiz ${ }^{1}$, Fevzi Oktay Şişman ${ }^{1}$, Beliz Koçyiğit ${ }^{1}$, Mehmet Gürkan Arıkan², Tevfik Aktoz ${ }^{2}$ \\ ${ }^{1}$ Trakya University School of Medicine, Edirne, TURKEY \\ ${ }^{2}$ Department of Urology, Trakya University School of Medicine, Edirne, TURKEY
}

\begin{abstract}
Aims: Horseshoe kidneys are the most common type of renal fusion anomaly and it may be associated with other anomalies and complications. Our case aims to present the partial nephrectomy of a horseshoe kidney with renal-cell carcinoma and cholecystectomy. Case Report: A sixty-year-old male patient was admitted to our outpatient clinic with a $3 \mathrm{~cm}$ suspicious mass in the horseshoe kidney that was detected during an attack of acute cholecystitis. Computed tomography revealed a heterogeneous hypodense lesion containing millimetric calcific foci of $35 \times 31 \times 33 \mathrm{~mm}$ in size at the ventral middle part of the right kidney. Partial nephrectomy was performed non-ischemically and then cholecystectomy was performed. Pathological examination revealed stage T1a clear cell renal carcinoma, WHO/ISUP Grade 2 with a negative surgical margin. During follow-up; urea, creatinine, and glomerular filtration rate were found to be normal. Conclusion: Horseshoe kidneys are fairly common among renal anomalies. Cholecystectomy following non-ischemic partial nephrectomy for a tumor in the horseshoe kidney is a rare case. Keywords: Horseshoe kidney, nephrectomy, cholecystectomy
\end{abstract}

\section{INTRODUCTION}

Horseshoe kidneys (HSK) are the most common renal fusion anomaly. It is seen in $0.25 \%$ of the population and is twice as common in men as in women (1). Horseshoe kidney is characterized by abnormalities in the kidney's position, rotation, and vascular supply. It has been found that functional renal masses fused with ureters on both sides of the vertebral column remain without crossing the renal hilum to the bladder (2). The position of the isthmus connecting the two renal masses may differ from case to case. It may be midline or asymmetrical, referred to as an asymmetrical horseshoe kidney. Seventy percent of the asymmetrical horseshoe kidneys are dominant, and $80 \%$ of the cases are composed of the renal parenchyma and the remainder of the fibrous band. Although fusion occurs in the lower pole in more than $90 \%$ of the cases, it may also occur in the upper pole in a small number of cases (2).

Horseshoe kidneys may also be present in $30 \%$ as an isolated condition, but there are different types of associated abnormalities (1). It mostly consists of lithiasis, ure- teropelvic obstruction, and infections. Additionally, there is a higher risk of kidney lesions and an increased incidence of malignancy in trauma. It is important for physicians to have knowledge of embryology and anatomy in order to understand the complications affecting HSK.

Horseshoe kidneys in adults are mostly asymptomatic. It can be detected by intravenous pyelography, ultrasound, or computed tomography (CT) scanning. Scintigraphy can also be used to detect HSK (3). In normal embryonic development, kidneys come out of the pelvis in the 7 th and 8 th weeks. In the case of HSK, the inferior mesenteric artery blocks the ascent of the kidney. Therefore it gets stuck in the mid-abdominal area due to not migrating out of the pelvis as it does in normal embryonic development (3).

Horseshoe kidneys can be associated with other anomalies. Common anomalies for this instance are congenital gastrointestinal, central nervous system, skeletal and chromosomal abnormalities. Glenn (4) reported a $78.9 \%$ lower incidence of associated congenital anomalies in stillborn fetuses and infants, $28.5 \%$ in children, and $3.5 \%$ in adults. 
Benign and malignant tumors are also associated with HSK. Malignancy is mostly attributed to teratogenic factors. Having HSK can increase the risk of kidney cancer. For example, the incidence of horseshoe kidneys in the general population is 1 in 400, a child with HSK has twice the risk of developing Wilms' tumor than the general population (5).

Our case presents a patient who underwent non-ischemic partial nephrectomy followed by cholecystectomy for horseshoe kidney malignancy. We believe this case report will give physicians more insight into this event.

\section{CASE REPORT}

A sixty-year-old male patient was admitted to our outpatient clinic with a $3 \mathrm{~cm}$ suspicious mass in the horseshoe kidney that was detected incidentally during an attack of acute cholecystitis two months ago. According to the detailed history of the patient, he was using oral anticoagulants due to coronary by-pass. The patient's blood pressure, heart rate and body temperature were normal and he did not have hematuria. On the other hand, he had gastrointestinal symptoms and weight loss.On physical examination, there was tenderness in the right upper quadrant of the abdomen, the other quadrants were found to be normal. There was no bilateral costovertebral angle sensitivity.

The routine hemogram, urine analysis, and serum electrolytes were normal. A horseshoe kidney was detected in the urinary system ultrasound, and a heterogeneous lesion of approximately $3 \mathrm{~cm}$ in size was observed on the right. CT revealed a horseshoe kidney and a heterogeneous hypodense lesion containing millimetric calcific foci of $35 \times 31 \times 33 \mathrm{~mm}$ in size at the ventral middle part of the right (Figure 1, 2, 3). In addition, the gallbladder wall was inflamed, and calculus was found in it (Figure 3, 4).

The operation was performed with an anterior subcostal incision. The Gerota's fascia was opened and the mass was reached directly. The operation was continued non-ischemically, and the mass was excised in accordance with oncological procedures. Then, cholecystectomy was performed by the general surgery team, and the gallbladder was excised without any complication. The postoperative follow-up was uneventful.

Histopathological examination revealed stage T1a clear cell renal carcinoma, WHO/ISUP Grade 2, and negative surgical margin. The histopathology of the gallbladder was reported as chronic cholecystitis. During follow-up; urea, creatinine, and glomerular filtration rate (GFR) were found to be normal.

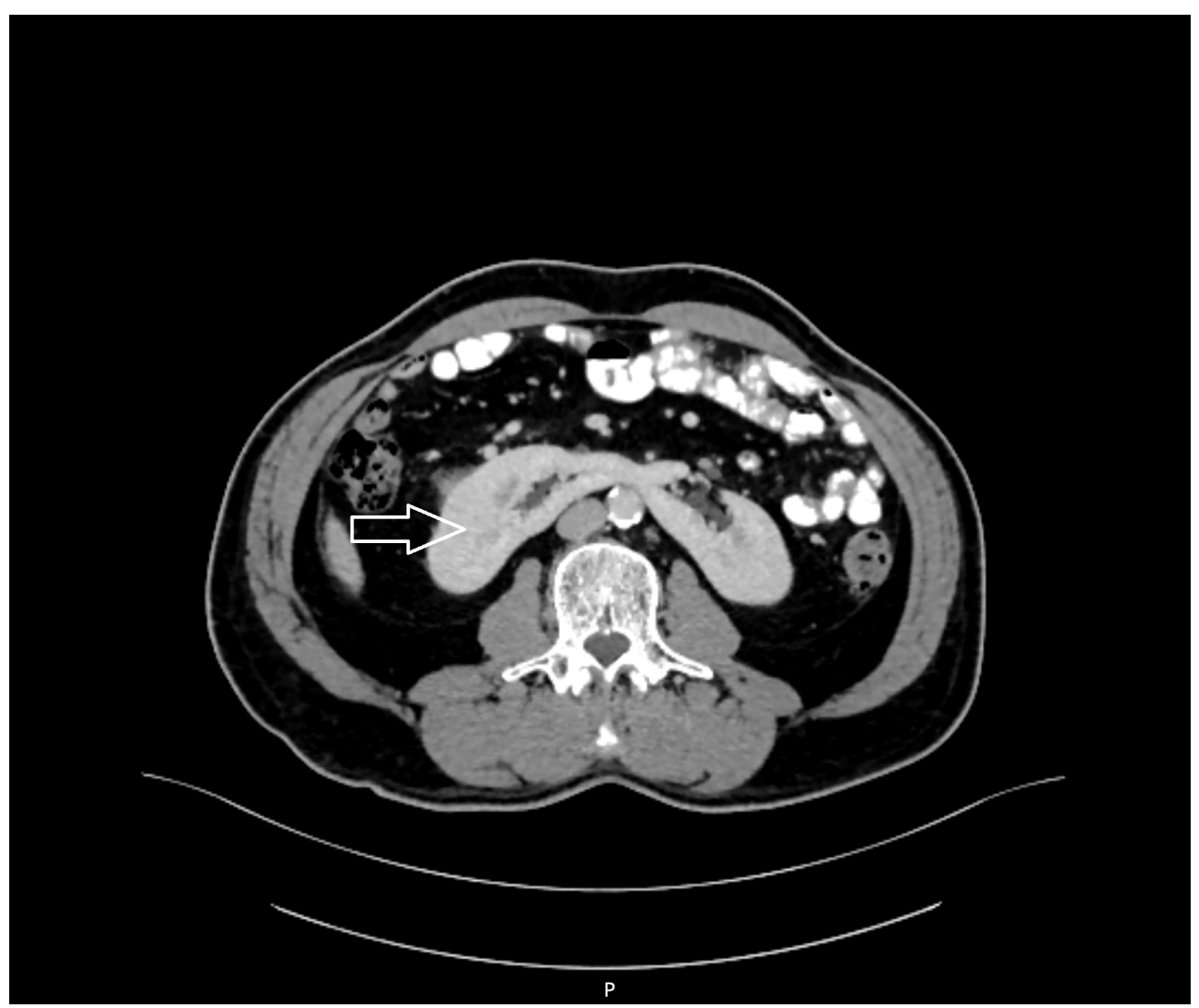

Figure 1: Transverse sectional view of the right horseshoe kidney on CT scan. 


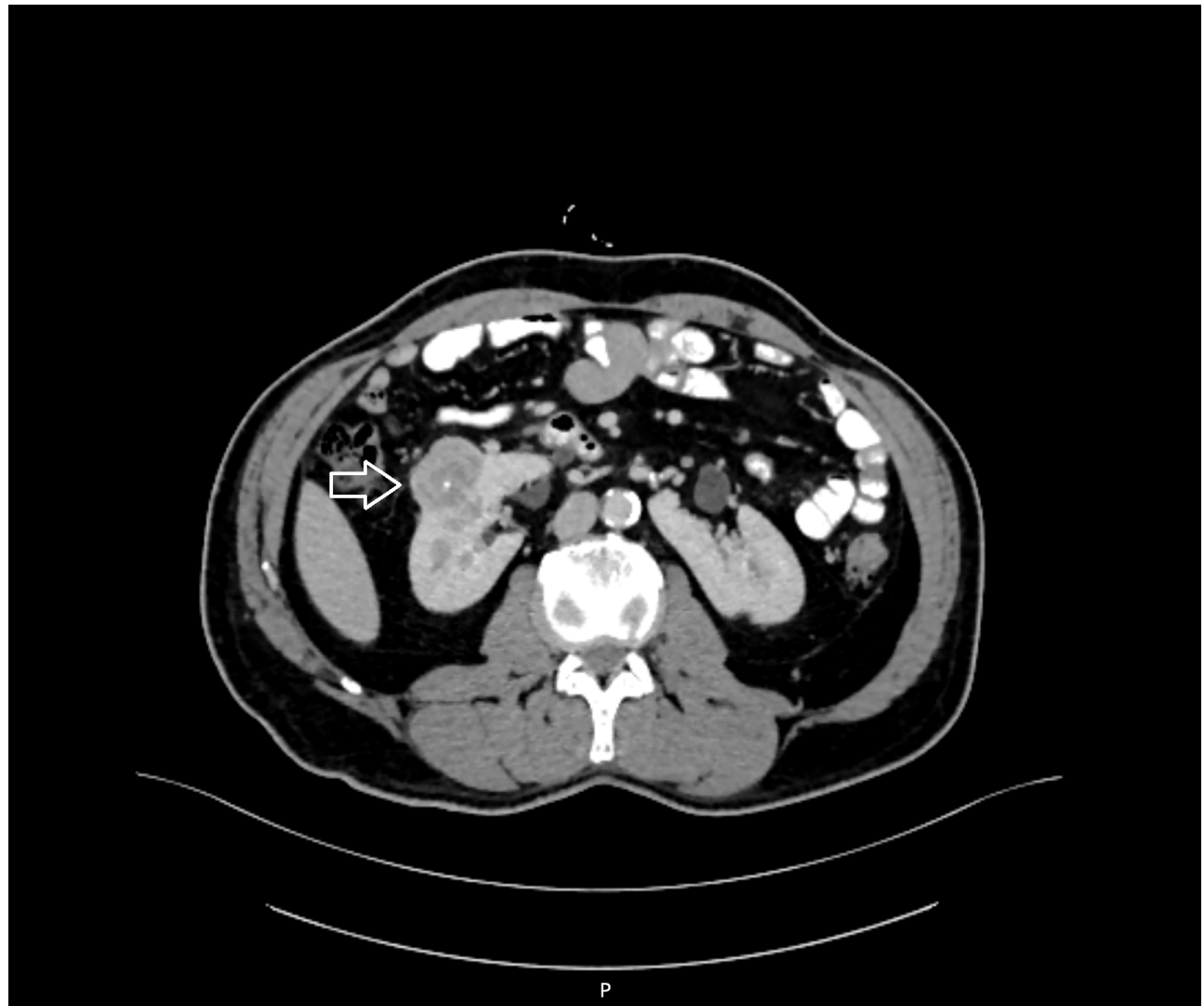

Figure 2: Transverse sectional view of the tumor in the right horseshoe kidney on CT scan.

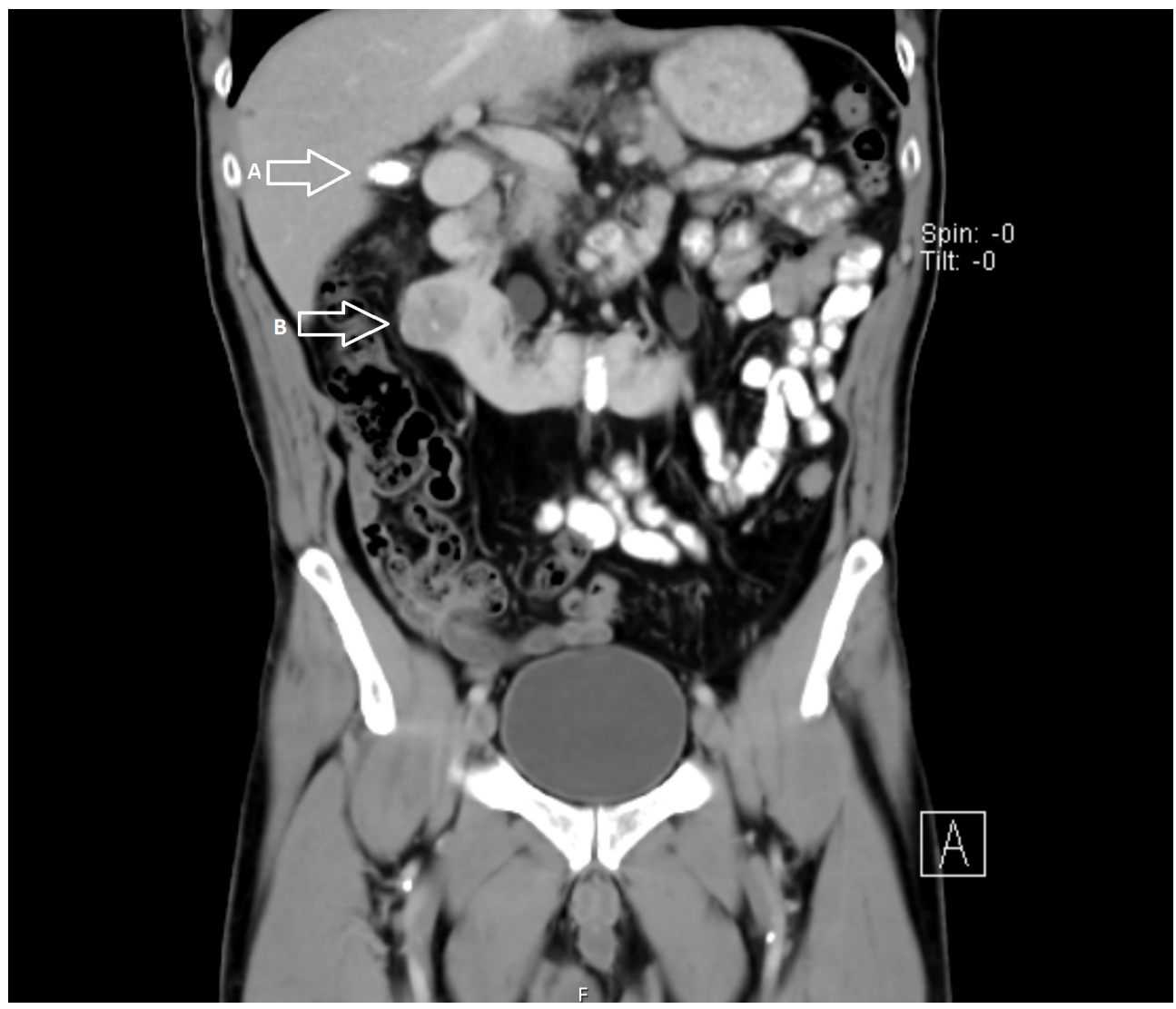

Figure 3: Coronal sectional view of the right horseshoe kidney and the gallbladder seen in the CT scan (A: calculus in the gallbladder and inflamed gallbladder wall B: tumor in the right horseshoe kidney). 


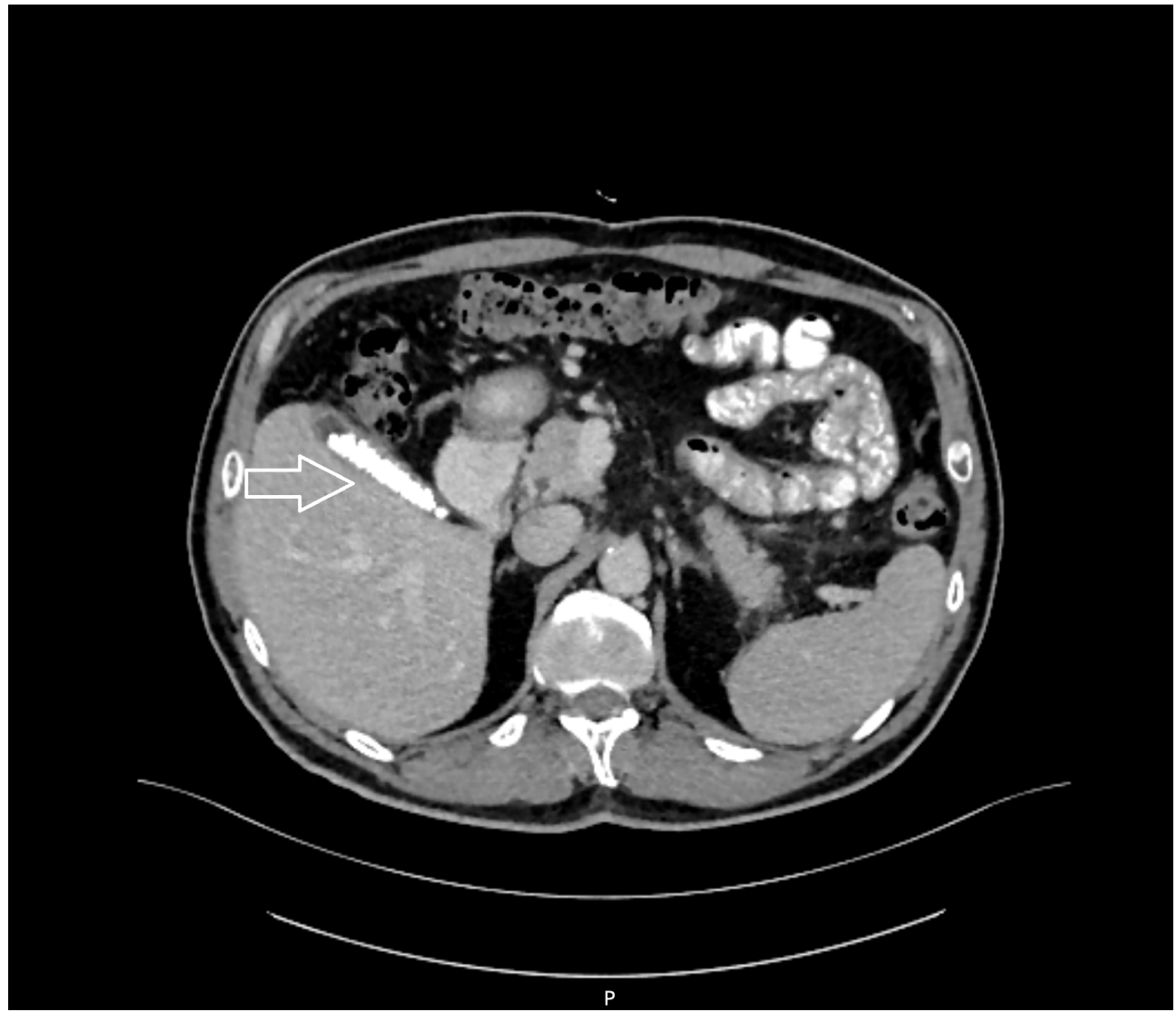

Figure 4: Transverse sectional view of the calculus in the gallbladder and inflamed gallbladder wall in the CT scan.

\section{DISCUSSION}

In our case, a sixty-year-old male patient was admitted to the hospital with an attack of acute cholecystitis, and a suspicious mass of $3 \mathrm{~cm}$ in the horseshoe kidney was found incidentally on CT. Bhandarkar et al. (6) stated that the probability of finding horseshoe kidneys by chance is approximately $50 \%$ of all cases.

Our case was diagnosed with horseshoe kidney incidentally which is similar to the case presented by Seker et al. (7). They presented a thirty-seven years old female patient incidentally diagnosed with HSK, similar to our case. Their patient had splenomegaly but after a CT scan, the patient had horseshoe kidney with a hypodense area $18 \times 12.5 \mathrm{~mm}$ in size at the left kidney. The patient was operated by left-open partial nephrectomy zero ischemia with a modified Chevron incision without complications during and after the procedure (7).

Tkocz et al. (8) had a patient with HSK with a tumor. The tumor was removed through a median incision after organ detachment. Cholecystectomy was performed as in our case, due to the presence of gall- bladder hydrocele.

The incidence of renal cell carcinoma in horseshoe kidneys is the same as in normal kidneys, and its development and course are not associated with the presence of the defect. According to the literature, transitional cell carcinoma is more common in horseshoe kidneys. This may be due to the presence of defects such as urinary obstruction, susceptibility to nephrolithiasis, and chronic infection $(9,10)$.

Ischemic segmental kidney necrosis due to insufficient collateral artery supply in HCC is a common complication. Ligation or division of an artery results in ischemic segmental renal necrosis (2). Justo-Janeiro et al. (11) had also operated on a HSK patient. Dissociation was by the ischemic line and total ischemic time was 125 minutes. However, in our case, the mass was excised non-ischemically. This is unusual for such a HSK surgery and there was no reduction in urea, creatinine, and GFR in postoperative follow-up. As a result, horseshoe kidneys are quite common among kidney anomalies. As mentioned earlier, the diagnosis of horseshoe kidneys is accidental in about $50 \%$ of all cases. 
As a conclusion, horseshoe kidneys are fairly common among renal anomalies. Cholecystectomy following non-ischemic partial nephrectomy for a tumor in the horseshoe kidney is a rare case. We believe that this case report will provide physicians a different perspective and additional information on the literature.

Ethics Committee Approval: N/A

Informed Consent: Informed verbal consent was obtained from the patient for this study.

Conflict of Interest: The authors declared no conflict of interest. Author contributions: Concept: EC, FOŞ, BK, MGA, TA Design: EC, FOŞ, BK, MGA, TA Supervision: EC, FOŞ, BK, MGA, TA Materials: EC, FOŞ, BK, MGA, TA Data collection and/or processing: EC, FOŞ, BK, MGA, TA Analysis and/or Interpretation: EC, FOŞ, BK, MGA, TA Literature Search: AG, SA, TA Writing Manuscript: EC, FOŞ, BK, MGA, TA Critical Review: EC, FOŞ, BK, MGA, TA. Financial disclosure: The authors declared that this study received no financial support.

Editor-in-chief's Note: Three of the authors of this article, Elif Cengiz, Fevzi Oktay Şişman, Beliz Koçyiğit are members of the editorial board of Turkish Medical Student Journal. However, they did not take place at any stage of the editorial decision of the manuscript. The editors who evaluated this manuscript are from other institutions.

\section{REFERENCES}

1. Schiappacasse G, Aguirre J, Soffia P et al. CT findings of the main pathological conditions associated with horseshoe kidneys. Br J Radiol 2015;88(1045):20140456.

2. Kirkpatrick JJ, Leslie SW. Horseshoe Kidney. [Updated 2020 Aug 12]. In: StatPearls [Internet]. Treasure Island (FL): StatPearls Publishing; 2020 Jan-. Available from: URL: https://www.ncbi.nlm.nih. gov/books/NBK431105/.

3. Natsis K, Piagkou M, Skotsimara A et al. Horseshoe kidney: a review of anatomy and pathology. Surg Radiol Anat 2014;36(6):51726.

4. Glenn JF. Analysis of 51 patients with horseshoe kidney. N Engl Med 1959;261:684-7.

5. Mesrobian HJ, Kelalis PP, Hrabovsky E et al. Wilms tumor in horseshoe kidneys: a report from the National Wilms Tumor Study. J Urol 1985;133:1002-3.

6. Bhandarkar KP, Kittur DH, Patil SV et al. Horseshoe kidney and associated anomalies: single institutional review of 20 cases. Afr J Paediatr Surg 2018;15(2):104-7.

7. Şeker KG, Şam E, Şahin S et al. Partial nephrectomy in horseshoe kidney: primary carcinoid tumor. Arch Ital Urol Androl 2017;89(4):316-8.

8. Tkocz M, Kupajski M. Tumour in horseshoe kidney - different surgical treatment shown in five example cases. Contemp Oncol 2012;16(3):254-7.

9. Hohenfellner M, Schultz-Lampel D, Lampel A et al. Tumor in horseshoe kidney: clinical implications and review of embryogenesis. J Urol 1992;147:1098-102.

10. Rubio Briones J, Regalado Pareja R, Martin FS et al. Incidence of tumoural pathology horseshoe kidneys. Eur Urol 1998;33:175-9. 11. Justo-Janeiro JM, Orozco EP, Reyes FJ et al. Transplantation of a horseshoe kidney from a living donor: case report, long term outcome and donor safety. Int J Surg Case Rep 2015;15:21-5. 